\title{
Knowledge Regarding Glasgow Coma Scale among Nurses Working at Selected Hospitals of Chitwan, Nepal
}

\author{
Prativa Sedain, ${ }^{1}$ Mina Kumari Bhusal ${ }^{2}$ \\ ${ }^{1}$ Department of ENT, B.P. Koirala Memorial Cancer Hospital, Bharatpur, Chitwan, Nepal, ${ }^{2}$ Bharatpur Hospital Nursing \\ College, Bharatpur-10, Chitwan.
}

\begin{abstract}
Background: Glasgow Coma Scale (GCS) refers to the internationally standardized measurement tool used to check the level of consciousness. The objective of this study was to assess the knowledge regarding Glasgow Coma Scale among nurses working in selected Hospital of Bharatpur, Chitwan. Methods: A descriptive cross-sectional study was conducted among 154 nurses working in the different critical wards (like ICU, CCU, NICU) of Bharatpur using convenient sampling. Data was collected by using structured, self -administered questionnaire. Results: The mean age of the nurses was 23.24 years. Majority belongs to age group 20-24 years (72.1\%), Proficiency Certificate Level Nursing (69.5\%), education in private institution $(90.9 \%)$, staff nurse $(89.6 \%),<12$-month experience (working in that unit) $(58.9 \%)$, receive in-service education (professional training or staff development program) $(57.8 \%)$. Only $33.1 \%$ of the nurse had good level of knowledge. Likewise, $66.9 \%$ nurses had good knowledge regarding eye-opening component, $33.0 \%$ had good knowledge regarding motor component and $66.2 \%$ had good knowledge regarding verbal response of GCS. The statistical significant influencing variable for the level of knowledge are institute of getting education ( $\mathrm{p}=0.028)$, availability of protocol on GCS $(\mathrm{p}=0.048)$ and habit of self-directed learning $(\mathrm{p}=0.036)$. Conclusions: It is concluded that majority of nurses have unsatisfactory level of knowledge regarding GCS.
\end{abstract}

Keywords: Glasgow Coma Scale; nurses; Nepal.

\section{INTRODUCTION}

The Glasgow Coma Scale (GCS), designed in 1974, is a tool developed by Graham Teasdale and Bryan J. Jennett, to communicate the level of consciousness of patients with acute or traumatic brain injury. It is the gold standard used for all acute medical and trauma patients. It is used to identify neurologic dysfunction and follow-up progress of level of consciousness, predict prognosis and standardize communication among health professionals which tested three neurological aspects of the patient's response: eye-opening, limb movement, and vocalization. It is obtained by observation of spontaneous activities and use of verbal and/or painful stimulus. ${ }^{1}$ It is therefore, the most sensitive and reliable indicator of all neurological patients. It is specific and structured, allowing health care professionals to arrive at the same conclusion regarding the patient's status. ${ }^{2}$

In a Glasgow Coma Scale, patient is assessed against the criteria of the scale, and the resulting points give a patient score between 3 to 15 , where 3 indicate deep unconsciousness, score less than 8 indicates coma and 15 indicates fully awareness. ${ }^{4}$ Persons with GCS scores of 3 to 8 are classified with a severe traumatic Brain Injury (TBI) or comatose, those with scores of 9 to 12 are classified with a moderate TBI, and those with scores of 13 to
15 are classified with a mild TBI. ${ }^{5}$ GCS Scale, identify changes to consciousness in traumatic brain injury patients, is a tool that requires nurses to fully understand its purpose and how to use it. Identifying the patients that require scoring is the first step in properly using the scale. Nurses who work in areas that care for these patients need to be competent in assessing GCS.

The scoring will detect early deterioration in such patients showed that initial assessment of GCS obliviated unnecessary diagnostic tests and treatments. ${ }^{10}$ In the context of Nepal, only a few articles are related to this topic. The main objective of this study to assess the level of knowledge on each component of the Glasgow coma scale and association between levels of knowledge on GCS with other variables.

\section{METHODS}

A cross-sectional study was conducted to assess the knowledge regarding the Glasgow coma scale among Nurses of Bharatpur Hospital, and College of medical sciences in the ICU, Neuro ward, post-operative ward and Emergency ward from July to August 2018. Sample size was calculated based on study conducted by P. K. Sherin in Lucknow showed that $10.01 \%$ had good

Correspondence: Ms. Prativa Sedain, Department of ENT, B.P. Koirala Memorial Cancer Hospital, Bharatpur, Chitwan, Nepal. Email: prativa.sedain@gmail.com. Phone:+977-9845181464. Article received: 2019-05-21. Article accepted: 2019-11-25. 
knowledge on GCS. ${ }^{10}$ Sample size was calculated using, $(\mathrm{n})=\mathrm{Z}^{2} \mathrm{pq} / \mathrm{e}^{2}=\left(1.96^{2 *} 0.1001 * 0.8999\right) /\left(0.05^{2}\right)=$ 139 , using $5 \%$ permissible error and Z-score value at $95 \% \mathrm{CI}$, as 1.96 . The optimal sample size for this study was 139 . By using $10 \%$ as non-response error the total sample was $139+15=154$. Convenient sampling was used to select the nurses. SelfStructure questionnaire was used to collect the data. Reliability of the instrument was determined by using Cronbach's alpha using SPSS which gives the value as 0.78 . Pretesting was done at the ICU of Chitwan Medical College teaching Hospital. Ethical approval was obtained from Bharatpur Hospital and permission for data collection was obtained from the College of Medical Sciences Teaching Hospital.

The collected data was checked daily before leaving to ensure the completeness of the information. The collected data was serially compiled in the file to prevent loss and damage. After data collection, the questionnaire was rechecked for accuracy, utility, and completeness. Serial numbers were given for each question. On completion of proper editing the data was arranged a homogenous group based on their characteristics for use in the description and analysis of the study result. Each data was coded after the data had been gathered. The correct response was coded 1 and incorrect response was coded 0. Data was entered in EpiData 3.1 and data analysis was done using SPSS-17. Data was analyzed by using descriptive and inferential statistics. Similarly, in descriptive statistics result was presented in the table, figure, frequency, percent, mean, standard deviation. In inferential statistics to find the association between the level of knowledge with other related variables, Chi-square test was used.

Level of knowle dge: Knowledge of GCS is defined in this study as the ability of nurses to elicit correct responses to 23 questions on GCS knowledge as contained in the instrument. The knowledge maximum score is 23 . A score range of 1-7 is rated poor knowledge, 8-16 average knowledge, and 1723 good knowledge of GCS. For each component of GCS $(\leq 33 \%)$ poor knowledge, (34-74\%) average knowledge and $(\geq 75 \%)$ good knowledge. ${ }^{16}$

\section{RESULTS}

Among 154 nurses, the majority $(72.1 \%)$ were in the 20-24 years age group. The mean $\pm \mathrm{SD}$ of age was $23.24 \pm 2.66$ years. Similarly, $87.7 \%$ of the nurses were residing in urban and $51.9 \%$ were Brahmin. Most of the, $87 \%$ of nurses were Hindu and $64.9 \%$ were unmarried. Majority $(69.5 \%)$ of nurses had completed PCL Nursing, (90.9\%) of nurses had attended private institution, most of the $(89.6 \%)$ nurses had less than or equal to 3 years experience, $53.2 \%$ of the nurses were working in Intensive Care Unit (ICU). Likewise, the majority
$(74.0 \%)$ of the nurses worked less than or equals to one year in critical care unit, $57.8 \%$ of nurses have not participated in in-service education regarding GCS. Likewise, most of the nurses $(81.8 \%)$ reported that they had a protocol regarding GCS in their working area and $88.3 \%$ of the nurses had habit of self- directed learning (Table 1).

\begin{tabular}{|c|c|c|}
\hline Characteristics & Number & Percentage \\
\hline \multicolumn{3}{|l|}{ Age (in years) } \\
\hline $20-24$ & 111 & 72.1 \\
\hline $25-29$ & 39 & 25.3 \\
\hline $30-34$ & 4 & 2.6 \\
\hline Mean \pm SD & \multicolumn{2}{|c|}{$23.24 \pm 2.66$ Years } \\
\hline \multicolumn{3}{|l|}{ Residence } \\
\hline Urban (Municipality) & 135 & 87.7 \\
\hline Rural(VDC) & 19 & 12.3 \\
\hline \multicolumn{3}{|l|}{ Ethnicity } \\
\hline Bhramin & 80 & 51.9 \\
\hline Other caste & 74 & 48.1 \\
\hline \multicolumn{3}{|l|}{ Religion } \\
\hline Hindu & 134 & 87 \\
\hline Buddhist and others & 20 & 13 \\
\hline \multicolumn{3}{|l|}{ Marital status } \\
\hline Married & 54 & 35.1 \\
\hline Unmarried & 100 & 64.9 \\
\hline \multicolumn{3}{|l|}{ Qualification } \\
\hline PCL Nursing & 107 & 69.5 \\
\hline B.N./Bsc. Nursing & 47 & 30.5 \\
\hline \multicolumn{3}{|l|}{ Educational institute } \\
\hline Govemment & 14 & 9.1 \\
\hline Private & 140 & 90.9 \\
\hline \multicolumn{3}{|c|}{ Nursing working Experience } \\
\hline <3 Year & 138 & 89.6 \\
\hline 3 Year & 16 & 10.4 \\
\hline \multicolumn{3}{|l|}{ Working unit } \\
\hline ICU & 82 & 53.2 \\
\hline Post operative & 29 & 18.8 \\
\hline Emergency & 16 & 10.4 \\
\hline Neuro ward & 27 & 17.5 \\
\hline \multicolumn{3}{|l|}{ Duration of work time } \\
\hline$<1$ Year & 114 & 74 \\
\hline$\geq 1$ Year & 40 & 26 \\
\hline \multicolumn{3}{|c|}{ In-service education in working place } \\
\hline Yes & $65^{\circ}$ & 42.2 \\
\hline No & 89 & 57.8 \\
\hline \multicolumn{3}{|c|}{ Protocol of GCS in working place } \\
\hline Yes & 126 & 81.8 \\
\hline No & 28 & 18.2 \\
\hline \multicolumn{3}{|c|}{ Self- directed knowledge on GCS scale } \\
\hline Yes & 136 & 88.3 \\
\hline No & 18 & 11.7 \\
\hline
\end{tabular}

About (93.5\%) nurses know the definition of GCS and $91.6 \%$ nurses know the component of GCS. Most of the nurses $(82.5 \%)$ know the eye-opening component score, $81.8 \%$ nurses know the verbal response component score and $83.1 \%$ know the motor response component score. Also, 49.4\% 
nurses know that the cranial nerve responsible for eye movement and opening of eyelid and majority of the nurses $(76.0 \%)$ know that the GCS score for the eye-opening if the patient opens eye only on pain stimuli. Similarly, most of the nurses $(78.6 \%)$ know GCS score if patient opens eye only on verbal command, $79.2 \%$ nurses know GCS score for patient whose eyes are closed because of facial injury or swelling and $81.8 \%$ of the nurses answered correctly that the area of orientation that moderate traumatic brain injury (Table 2). Out of 154 nurses, $66.9 \%$ of the nurse had good knowledge, $11.7 \%$ of the nurses had average level of knowledge and only $21.4 \%$ had poor level of knowledge regarding eye-opening component of GCS. Among all, $(37.0 \%)$ had a good knowledge, $42.9 \%$ had an average knowledge and $20.1 \%$ had a poor knowledge regarding motor components of GCS. Also, $66.2 \%$ of nurses had a good knowledge, $16.2 \%$ had an average knowledge and only $17.5 \%$

\begin{tabular}{|c|c|c|}
\hline Components of GCS & Number & Percent \\
\hline Definition of GCS & 144 & 93.5 \\
\hline Component of GCS & 141 & 91.6 \\
\hline Eye opening component score & 127 & 82.5 \\
\hline Verbal response component score & 126 & 81.8 \\
\hline Motor response component score & 128 & 83.1 \\
\hline $\begin{array}{l}\text { GCS score if patient opens eyes only after verbal command, uses inappropriate word and } \\
\text { localizes the area of pain stimuli }\end{array}$ & 90 & 58.4 \\
\hline Appropriate body part nurse should check first for painfuls stimuli to patient & 41 & 26.6 \\
\hline Cranial nerve responsible for eye movement and opening of eye lid & 76 & 49.4 \\
\hline GCS score for the eye opening if patient opens eye only on pain stimuli & 117 & 76.0 \\
\hline GCS score for eye opening if patient opens eye only on verbal command & 121 & 78.6 \\
\hline GCS score for patient whose eyes are closed because of facial injury or s welling & 122 & 79.2 \\
\hline Area ofbrain responsible forspeech control & 77 & 50.0 \\
\hline GCS score for verbal response if patient use inappropriate words with no sustain sentences & 119 & 77.3 \\
\hline Areas of orientation that nurse should assess for verbal response & 126 & 81.8 \\
\hline Interpretation of incomprehensible sounds in verbal response of GCS score & 107 & 69.5 \\
\hline Verbal response for patient having ET tube/ Tracheos tomy tube & 25 & 16.2 \\
\hline GCS score for motor response if the patient localizes the painfulstimuli & 96 & 62.3 \\
\hline GCS score for motor response if the patient obeys verbal command & 108 & 70.1 \\
\hline GCS score represents Comatose patients & 30 & 19.5 \\
\hline GCS that indicates critical situation and examiner should be alert & 74 & 48.1 \\
\hline GCS interval indicates moderate severity in patient condition) & 97 & 63.0 \\
\hline GCS interval indicate improved patient condition & 112 & 72.7 \\
\hline
\end{tabular}

nurse should assess for verbal response. Only $62.3 \%$ of the nurse know that the GCS score for motor response if the patient localizes the painful stimuli, $70.1 \%$ of the nurses know the GCS score for motor response if the patient obey verbal command and $19.5 \%$ nurses answered correctly concerning for GCS score represents comatose patients, $63.0 \%$ of participants answered as correct, indicating this interval between score 12-9 for

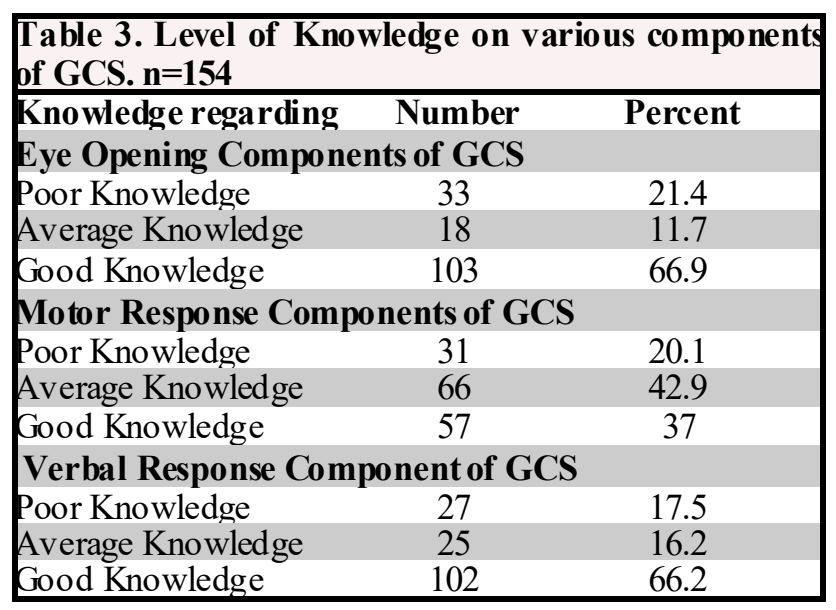

had a poor knowledge regarding verbal components of GCS ( Table 3). This research showed that $33.1 \%$ of the nurse had a good level of knowledge, $51.3 \%$ of the nurse had an average level of knowledge and only $15.6 \%$ had a poor level of knowledge regarding GCS. The overall mean \pm SD score of knowledge regarding the level of knowledge on GCS was 14.94 \pm 4.22 (Figure 1).

Institute of getting education ( $\mathrm{p}=0.028)$, availability

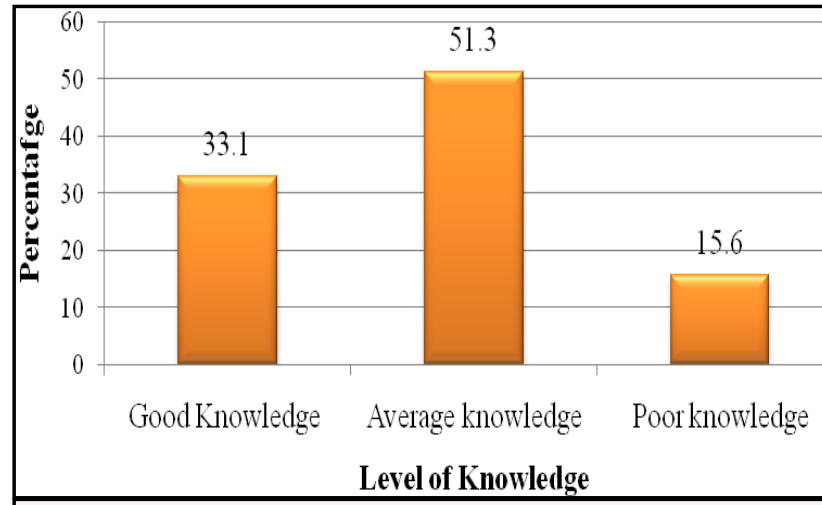

Figure 1. Overall level of knowledge on Glasgow coma Scale (GCS). $n=154$ 
Sedain et al. Knowledge Regarding Glasgow Coma Scale (GCS) among Nurses..

of protocol regarding GCS in the work setting $(p=0.048)$ and habit of self-directed knowledge $(p=0.036)$ are the statistically significant variables, with knowledge of GCS and all other variables such as qualification, work unit, experience, in-service education were statically insignificant (Table 4).
14.94 \pm 4.22 . A research study conducted by Ehwarieme \& Anarado showed that $41.7 \%$ of the nurses had good, 25.2\% average and 33.0\% had poor knowledge on the GCS. ${ }^{8}$ Singh research showed $2.96 \%$ had good knowledge, $41.48 \%$ had satisfactory knowledge, and $55.56 \%$ had poor knowledge on GCS. ${ }^{13}$ Teles et al., found that

\begin{tabular}{|c|c|c|c|c|}
\hline Variables & Poor knowledge n(\%) & Average knowledge n(\%) & Good Knowledge n(\%) & p-value \\
\hline \multicolumn{5}{|c|}{ (1) } \\
\hline$<25$ & $17(15.3)$ & $59(53.2)$ & $35(31.5)$ & \multirow{2}{*}{0.74} \\
\hline$\geq 25$ & $7(16.3)$ & $20(46.5)$ & $16(37.2)$ & \\
\hline \multicolumn{5}{|l|}{ Residence } \\
\hline Urban & 19(14.1) & $67(49.6)$ & $49(36.3)$ & \multirow{3}{*}{0.63} \\
\hline Rural & $5(26.3)$ & $12(63.2)$ & $2(10.5)$ & \\
\hline \multicolumn{4}{|l|}{ Ethnicity } & \\
\hline Bhramin & $10(12.5)$ & $44(55)$ & $26(32.5)$ & \multirow{2}{*}{0.64} \\
\hline Other caste & 14(18.391) & $35(47.29)$ & $25(33.78)$ & \\
\hline \multicolumn{4}{|l|}{ Religion } & \multirow{4}{*}{0.834} \\
\hline Hindu & $20(14.9)$ & $69(51.5)$ & $45(33.6)$ & \\
\hline Buddhist & $4(20.0)$ & $10(50.0)$ & $6(30.0)$ & \\
\hline \multicolumn{4}{|l|}{ Marital status } & \\
\hline Married & $11(20.4)$ & $23(42.6)$ & $20(37.0)$ & \multirow{3}{*}{0.242} \\
\hline Unmarried & $13(13.0)$ & $56(56.0)$ & $31(31.0)$ & \\
\hline \multicolumn{4}{|l|}{ Qualification } & \\
\hline PCL Nursing & $21(19.6)$ & $53(49.5)$ & $33(30.8)$ & \multirow{3}{*}{0.19} \\
\hline BN/B.SC Nursing & $3(6.4)$ & $26(55.3)$ & $18(38.3)$ & \\
\hline \multicolumn{4}{|c|}{ Institute of getting education } & \\
\hline Govemment & $6(42.9)$ & $4(28.6)$ & $4(28.6)$ & \multirow[b]{2}{*}{$0.028^{\mathrm{a}}$} \\
\hline Private & $18(12.9)$ & $75(53.6)$ & $47(33.6)$ & \\
\hline \multicolumn{4}{|c|}{ Working Experience in nursing field } & \multirow{4}{*}{0.24} \\
\hline$<3$ Year & $21(15.2)$ & $73(52.9)$ & $44(31.9)$ & \\
\hline$\geq 3$ Year & $3(18.8)$ & $6(37.5)$ & $7(43.8)$ & \\
\hline \multicolumn{4}{|l|}{ Work unit } & \\
\hline ICU & $16(19.5)$ & $39(47.6)$ & $27(32.9)$ & \multirow{5}{*}{$0.363^{\mathrm{a}}$} \\
\hline Post-operative & $4(13.8)$ & $19(65.5)$ & $6(20.7)$ & \\
\hline Emergency & $1(6.3)$ & $9(56.3)$ & $6(37.5)$ & \\
\hline Neuro ward & $3(11.1)$ & $12(44.4)$ & $12(44.4)$ & \\
\hline \multicolumn{4}{|l|}{ Present work time } & \\
\hline$<1$ Year & $16(14.0)$ & $58(50.9)$ & $40(35.1)$ & \multirow{3}{*}{0.547} \\
\hline$\geq 1$ Year & $8(20.0)$ & $21(52.5)$ & $11(27.5)$ & \\
\hline \multicolumn{4}{|c|}{ In-service education in working place } & \\
\hline Yes & $9(13.8)$ & $37(56.9)$ & $19(29.2)$ & \multirow{3}{*}{0.491} \\
\hline No & $15(16.9)$ & $42(47.2)$ & $32(36.0)$ & \\
\hline \multicolumn{4}{|c|}{ Protocol of GCS in working place } & \\
\hline Yes & $19(15.1)$ & $58(46.0)$ & 49(38.9) & \multirow{3}{*}{0.048} \\
\hline No & $5(17.9)$ & $21(75.0)$ & $2(7.1)$ & \\
\hline \multicolumn{4}{|c|}{ Self- directed knowledge on GCS scale } & \\
\hline Yes & $18(13.2)$ & $70(51.5)$ & $48(35.3)$ & \multirow{2}{*}{0.036} \\
\hline No & $6(33.3)$ & $9(50.0)$ & $3(16.7)$ & \\
\hline
\end{tabular}

\section{DISCUSSION}

This study is focued on the nurse's knowledge regarding GCS and identifies factors associated with their knowledge. This research showed that 33.1\% of nurse a had good level of knowledge, $51.3 \%$ had an average level of knowledge and $15.6 \%$ had a poor level of knowledge regarding GCS. The overall mean $\pm \mathrm{SD}$ of knowledge score on GCS was
$74.55 \%$ of the staff nurses had average knowledge and $25.45 \%$ had poor knowledge in GCS, ${ }^{14}$ whereas Jaddoua et al. found that all nurses had inadequate knowledge in GCS. ${ }^{15}$ The research conducted by Ehwarieme showed that (41.7\%) of the respondents had good knowledge, $25 \%$ had moderate knowledge and $33.3 \%$ had poor knowledge on the GCS. ${ }^{16}$ The findings of Jaddoua et al., showed that 
nurses have inadequate in knowledge in all the 25 items of GCS. ${ }^{17}$ Our finding showed that, $37.0 \%$ had good knowledge regarding motor components of GCS and $66.9 \%$ had good knowledge regarding verbal components of GCS. Almost all nurses had adequate knowledge concerning application of GCS. ${ }^{11}$ Many nurses may know about the basic theoretical concepts of the GCS, however they are not able to apply that basic knowledge in clinical scenarios $62.6 \%$ of the participants in this study demonstrated good knowledge about basic concepts of the GCS only $5.2 \%$ of the participants had good knowledge on application of the basic knowledge in clinical situations. ${ }^{12}$ In a study of Santosh showed $39.4 \%$ nurses had good knowledge regarding eye-opening component. ${ }^{9}$ Decrease in percentage of good level of knowledge may be because a majority of the nurses were PCL nurse in compassion to Bachelor level in nursing. This study found a significant association between participants'gender and their knowledge of the GCS, with female nurses demonstrating statistically significantly higher levels of knowledge than male nurses. ${ }^{12}$ This study findings showed that level of knowledge regarding GCS is statistically significant with educational institute ( $\mathrm{p}$-value $=0.028$ ), availability of protocol $(\mathrm{p}$-value $=0.048)$ and habit of self-directed knowledge ( $\mathrm{p}$-value $=0.036$ ). There is no significant association between level of knowledge and socio-demographic data. ${ }^{8}$ There is a statistically significant association between knowledge and education level. ${ }^{13}$

\section{CONCLUSIONS}

GCS is an important tool used in monitoring all categories of patients with acute or traumatic brain injury either in detecting or improvement in their condition, based upon various literatures and this study finding showed that many of the nurses working in the critical care unit has lack of adequate knowledge on the tool. In the context of Nepal in order to make this profession strong, there should be more structured and detailed approach that should be planed to teach the skill along with demonstrations regarding GCS. So, this study concludes that a well-developed GCS training program, development of GCS protocol in the working unit and distribution of designated booklet on GCS to all nurses who were working in neurological wards help to increase their applied knowledge and improving the quality of nursing care.

\section{Limitations of the study}

In this study only a few numbers of nurses of Bharatpur were selected. Future researcher can conduct this study on a large scale with probability sampling.

\section{ACKNOWLEDGEMENTS}

We are thankful to all the nursing staff for their valuable support and time of data collection.

Conflict of interest: None

\section{REFERENCES}

1. Singh B, Chong MC, Zakaria MI, Cheng ST, Tang LY, Azahar NH. Assessing nurse's knowledge of Glasgow Coma Scale in emergency and outpatient department. Nursing research and practice. 2016;(5).

2. Palmer R, Knight J. Assessment of altered conscious level in clinical practice. British journal of nursing. 2006 Dec 14;15(22):1255-9.

3. Al-Quraan H, AbuRuz ME. Assessment Of Jordanian Nurses' Knowledge To Perform Glasgow Coma Scale. European Scientific Journal. 2016 Sep 1;12(27).

4. Ratcliff JJ, Adeoye O, Lindsell CJ, Hart KW, Pancioli A, McMullan JT, Yue JK, Nishijima DK, Gordon WA, Valadka AB, Okonkwo DO. ED disposition of the Glasgow Coma Scale 13 to 15 traumatic brain injury patients: analysis of the Transforming Research and Clinical Knowledge in TBI study. The American journal of emergency medicine. 2014 Aug 1;32(8):84450.

5. Weir CJ, Bradford AP, Lees KR. The prognostic value of the components of the Glasgow Coma Scale following acute stroke. Qjm. 2003 Jan 1;96(1):67-74.

6. Hien NT. The accuracy of Glasgow coma scale knowledge and performance among Vietnamese nurses (Doctoral dissertation).-2011

7. Ehwarieme TA, Anarado AN. Nurses' knowledge of Glasgow coma scale in neurological assessment of patients in a selected tertiary hospital in Edo State, Nigeria. Africa journal of nursing and midwifery. 2016 Jan 1;18 (2):74-86.

8. Santos WC, Vancini-Campanharo CR, Lopes MC, Okuno MF, Batista RE. Assessment of nurse's knowledge about Glasgow coma scale at a university hospital. Einstein (São Paulo). 2016 Jun;14(2):213-8.

9. Jaddoua BA, Mohammed WK, Abbas AD. Assessment of nurse's knowledge concerning glasgow coma scale in neuro surgical wards. kufa Journal for Nursing sciences. 2013;3 (2):133-42.

10.Sherin PK. Staff Nurses Knowledge Regarding Glasgow Coma Scale in Selected Hospitals in Lucknow. 2019: july 2(7):115-16

11.Jaddoua BA, Mohammed WK, Abbas AD. Assessment of nurse's knowledge concerning glasgow coma scale in neuro surgical wards. kufa Journal for Nursing sciences. 2013;3 (2):133-42.

12.Alhassan A, Fuseini AG, Musah A. Knowledge 
of the Glasgow Coma Scale among Nurses in a Tertiary Hospital in Ghana. Nursing Research and Practice. 2019;2019.

13.Singh B, Chong MC, Zakaria MI, Cheng ST, Tang LY, Azahar NH. Assessing nurses knowledge of Glasgow Coma Scale in emergency and outpatient department. Nursing research and practice. 2016;2016.

14.Teles M, Bhupali P, Madhale M. Effectiveness of self instructional module on knowledge and skills regarding use of Glasgow coma scale in neurological assessment of patients among nurses working in critical care units of KLE Dr. Prabhakar Kore hospital and medical research centre, Belgaum. Journal of Krishna Institute of Medical Sciences University. 2013;2(1):98-104.
15.A. Jaddoua, W. K. Mohammed, and A. D. Abbas, "Asesment of Nurse's knowledge concerning glasgow coma scale in neuro surgical wards," Journal of Kufa for Nursing Scienc, vol. 3, pp. 133-142, 2013.

16.Ehwarieme TA, Anarado AN. Nurses' knowledge of Glasgow coma scale in neurological assessment of patients in a selected tertiary hospital in Edo State, Nigeria. Africa journal of nursing and midwifery. 2016 Jan 1;18 (2):74-86.

17.Jaddoua BA, Mohammed WK, Abbas AD. Assessment of nurse's knowledge concerning glasgow coma scale in neuro surgical wards. kufa Journal for Nursing sciences. 2013;3 (2):133-42.

Citation: Sedain P, Bhusal MK. Knowledge Regarding Glasgow Coma Scale (GCS) among Nurses working at selected hospitals of Chitwan, Nepal. JCMS Nepal. 2019; 15(4):276-81. 